\title{
Short-term proton pump inhibitor treatment may cause hypomagnesaemia in critically ill patients - a pilot study
}

\author{
Łukasz J. Krzych ${ }^{1}$, Piotr Łój ${ }^{1}$, Teresa Nowak², Wojciech Kazura² and Piotr Knapik ${ }^{4}$ \\ 'Department of Anaesthesiology and Intensive Care, School of Medicine in Katowice, Medical University of Silesia, Katowice, Poland; ${ }^{2}$ Students' \\ Scientific Society, Department of Anaesthesiology and Intensive Care, School of Medicine in Katowice, Medical University of Silesia, Katowice, \\ Poland; ${ }^{4}$ Department of Cardiac Anaesthesia and Intensive Therapy, School of Medicine with the Division of Dentistry in Zabrze, Medical Uni- \\ versity of Silesia in Katowice, Silesian Centre for Heart Diseases, Zabrze, Poland
}

\begin{abstract}
Many studies have suggested a link between long-term PPI treatment and hypomagnesaemia, though none of them investigated the short-term exposure in high-risk patients. We sought to investigate this issue in 90 critically ill patients. We assessed serum Mg concentrations, necessity of $\mathrm{Mg}$ supplementation, PPI dose, duration of PPI therapy and route of administration. In multiple analysis we found that $\mathrm{Mg}$ supplementation (positive effect $/ p=0.03$ ) and enteral route of PPI administration (negative effect $/ p=0.02$ ) had significant impact on $\mathrm{Mg}$ concentration. Although the deleterious relationship between short-term PPI treatment and Mg concentration was found, further studies should be provided to confirm this interesting effect.
\end{abstract}

Key words: proton-pump inhibitors, hypomagnesaemia, critically ill

Received: 12 January, 2017; revised: 28 February, 2017; accepted: 04 April, 2017; available on-line: 24 June, 2017

e-mail: Ikrzych@sum.edu.pl

Abbreviations: Mg, Magnesium; IPP, Proton-pump inhibitor; ICU, Intensive Care Unit; SAPS, Simplified Acute Physiology Score; CRRT, continuous renal replacement therapy

\section{INTRODUCTION}

Many studies have suggested a link between longterm PPI treatment and hypomagnesaemia (Park et al, 2014; Zipursky et al., 2014; Kieboom et al., 2015; Toh et al., 2015). This effect is more pronounced when the exposure lasts at least 6 months while PPI dose seems to be of low importance. PPI-induced hypomagnesaemia is thought to be caused by $\mathrm{Mg}$ intestinal malabsorption, probably due to inhibition of TRPM6 and -7 active transport channels (Bai et al., 2012).

PPIs are frequently used in ICU for stress ulcer prevention or treatment, although this indication still remains uncertain (Krag et al., 2016). Hypomagnesaemia in critically ill patients may induce potentially fatal complications, including hypoparathyroidism, ventricular arrhythmias, coronary artery vasospasm or even sudden cardiac death (Epstein et al., 2006; Hoorn et al., 2010; El-Charabaty et al., 2013). Therefore, we sought to verify the association between short-term treatment with PPI and serum $\mathrm{Mg}$ concentration in the ICU environment.

\section{MATERIALS AND METHODS}

This observational study included 90 consecutive patients hospitalized in the multidisciplinary ICU in 2015. Serum Mg concentrations, necessity of $\mathrm{Mg}$ supplemen- tation, PPI dose, duration of therapy and route of its administration were assessed. SAPS II score was calculated. The results were adjusted to the fact of CRRT use. Serum magnesium level was measured by colorimetric method based on reaction with xylidyl blue in an alkaline medium with addition of EGTA.

Patient confidentiality was ensured as the dataset was fully anonymised. Approval of the Ethics Committee was not necessary because the project was non-interventional and did not extend beyond routine laboratory sampling. Informed consent was obtained from conscious patients.

The statistical analysis was based on the procedures available in the licensed MedCalc (v14) software. Quantitative variables are presented as mean with standard deviation (those normally-distributed) or median with interquartile range (IQR) (skewed distribution). All variables were tested for normal distribution using the Shapiro-Wilk test. Qualitative variables are presented as percentage. Between-group differences were evaluated with the chi-squared test. Student's t-test or U Mann-Whitney test were used for quantitative data. Correlation was determined by the use of Spearman rank coefficient (R). Multiple regression was used to verify findings from bivariate analyses. The $p$-value $<0.05$ was considered statistically significant.

\section{RESULTS}

Baseline characteristics of the study group are listed in Table 1. Mean serum Mg concentration was $0.96 \pm 0.17$ $\mathrm{mmol} / \mathrm{l}$ and was higher in patients who underwent CRRT (CRRT+1.0 $\pm 0.17 \mathrm{mmol} / 1$ vs. CRRT $-0.94 \pm 0.16$ $\mathrm{mmol} / \mathrm{l} ; p=0.04) \mathrm{Mg}$ supplementation was necessary due to hypomagnesaemia in 50 subjects via a continuous infusion with mean dose of $2 \mathrm{~g}$ per day. Those subjects requiring $\mathrm{Mg}$ supplementation had higher $\mathrm{Mg}$ level (Sup$\mathrm{pl}+0.99 \pm 0.19 \mathrm{mmol} / 1$ vs. Suppl- 0.92 $\pm 0.13 \mathrm{mmol} / \mathrm{l}$; $p=0.04)$. PPIs were started on admission and used among all patients for 10 (IQR 5-21) days with a daily dose of 55 (IQR 40-70) $\mathrm{mg}$. None of subjects received H2-blockers, calcium channels blockers, $\beta$-blockers nor thiazides as potential confounders. No symptoms of hypomagnesaemia were present after the first dose of PPIs. We found that patients with PPIs given intravenously only $\left(\mathrm{PPI}_{\mathrm{IV}}\right)$ had higher $\mathrm{Mg}$ level compared to those who were treated intravenously and enterally $\left(\mathrm{PPI}_{\mathrm{PO}}\right)\left(\mathrm{PPI}_{\mathrm{IV}}\right.$ $1.02 \pm 0.17$ vs. $\mathrm{PPI}_{\mathrm{PO}} 0.93 \pm 0.16 ; p=0.02$ ) (Fig. 1). There was no correlation between $\mathrm{Mg}$ level and PPI dose (All: $\mathrm{R}=0.05 ; p=0.6 / \mathrm{PPI}_{\mathrm{IV}}: \mathrm{R}=-0.21 ; p=0.2 / \mathrm{PPI}_{\mathrm{PO}}: \mathrm{R}=0.05$; $p=0.7)$. No correlation was found between PPI therapy duration and $\mathrm{Mg}$ level $(\mathrm{R}=-0.11 ; p=0.3)$ as well. Mul- 
Table 1. Baseline characteristics of subjects

\begin{tabular}{lc}
\hline Variable & Value \\
\hline Demographics & 90 \\
\hline Number of patients & $66 \%$ \\
\hline Male gender & $64 \pm 13$ \\
\hline Age [years] & $53(39-67)$ \\
\hline SAPS II [points] & $19 \%$ \\
\hline Chronic obstructive pulmonary disease & $69 \%$ \\
\hline Coronary heart disease & $68 \%$ \\
\hline Arterial hypertension & $22 \%$ \\
\hline Diabetes mellitus & $40 \%$ \\
\hline Obesity & $12(6-27)$ \\
\hline ICU-related data & $41 \%$ \\
\hline Length of stay [days] & $5 \%$ \\
\hline Mortality & $5 \%$ \\
\hline Primary ICU admission diagnosis & $10 \%$ \\
\hline Respiratory failure & \\
\hline Multi-orgatory failure & $5 \%$ \\
\hline
\end{tabular}

ICU, intensive care unit; SAPS, Simplified Acute Physiology Score

tiple regression analysis proved that $\mathrm{Mg}$ supplementation $(p=0.03)$ and enteral route of PPI administration $(p=0.02)$ had statistically significant influence on $\mathrm{Mg}$ concentration (Table 2).

\section{DISCUSSION}

Up to our knowledge, this is the first investigation showing significant effect of short-term PPI use on $\mathrm{Mg}$ level in ICU patients. This interesting observation gives additional evidence that critically ill subjects should receive intravenous PPI treatment to prevent accidental hypomagnesaemia. Otherwise, enteral PPI treatment must be accompanied by $\mathrm{Mg}$ supplementation.

Molecular mechanisms of magnesium transporters action, including the $\mathrm{pH}$-dependent regulation of transient receptor potential melastatin transporters in the enterocytes, were proposed to explain the effect of PPI on magnesium reabsorption, but may be only a small part of a more complicated interplay of molecular biology, pharmacology, and genetic predisposition, especially in critically ill subjects with multiple comorbidities and poly-drug therapy (William et al., 2016).

Recent meta-analysis showed that long-term PPI use may increase the risk of hypomagnesaemia with

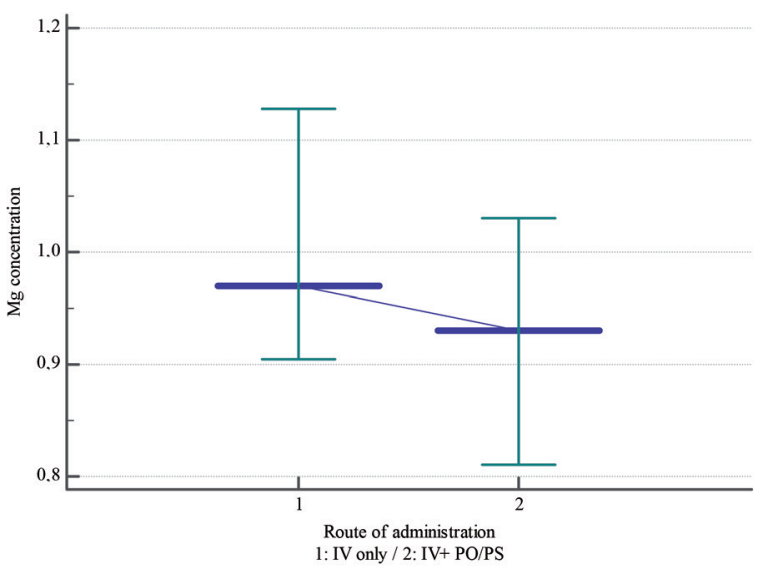

Figure 1. Serum magnesium concentration vs route of protonpump inhibitor administration

$\mathrm{OR}=1.775$ (95\%CI 1.077-2.924) (Park et al., 2014). However, significant heterogeneity among the included nine studies $(p<0.001)$ prevented the authors from reaching definitive conclusions. Due to novelty of our pilot study the comparability with other observations is limited. Danziger and coworkers (2016) in a single-centre observational study covering 11490 ICU patients found that the risk of hypomagnesaemia was $54 \%$ higher in patients who reported PPI use prior to admission but only if they were treated with diuretics.

One ought to remember that in critically ill patients PPI-induced hypomagnesaemia may be interfered by numerous confounders such as demographic data, use of loop diuretics, parenteral and enteral nutrition. Moreover, an attempt should be made to assess the impact of PPIs given prior to ICU admission. William and Danziger suggested that despite the increasing prevalence of this highly popular class of acid secretion inhibitors, and despite the potential significant risks associated with magnesium depletion, including cardiac arrhythmias and seizures, there are no well-designed studies to delineate the nature of this observed association (William et al., 2016). Taken together, it is necessary to verify the impact of short-term high-dose PPI use on $\mathrm{Mg}$ concentration in a prospective randomised manner.

\section{CONCLUSION}

Our preliminary data show that even short-term highdose enteral PPI treatment may cause hypomagnesaemia in critically ill patients.

\section{Conflicts of interests}

There are no conflicts of interests regarding this study.

Table 2. Multiple regression analysis for Mg concentration as the dependent variable

\begin{tabular}{|c|c|c|}
\hline Independent variables & Coefficient of regression \pm standard deviation & $P$ value \\
\hline Route of PPI administration & $-0.09 \pm 0.04$ & 0.02 \\
\hline PPI dose (per mg) & $0.0001 \pm 0.0001$ & 0.9 \\
\hline Need of Mg supplementation & $0.08 \pm 0.03$ & 0.03 \\
\hline CRRT use & $0.07 \pm 0.04$ & 0.06 \\
\hline
\end{tabular}

CRRT, continuous renal replacement therapy; PPI, proton-pump inhibitor 


\section{REFERENCES}

Bai JP, Hausman E, Lionberger R, Zhang X (2012) Modeling and simulation of the effect of proton pump inhibitors on magnesium homeostasis. 1. Oral absorption of magnesium. Mol Pharm 9: 3495$-505$

Danziger J, William JH, Scott DJ, Lee J, Lehman LW, Mark RG, Howell MD, Celi LA, Mukamal KJ (2013) Proton-pump inhibitor use is associated with low serum magnesium concentrations. Kidney Int 83: 692-699. doi: 10.1038/ki.2012.452

El-Charabaty E, Saifan C, Abdallah M, Naboush A, Glass D, Azzi G, Azzi Y, Khan A, Baydoun H, Rondla C, Parekh N, El-Sayegh S (2013) Effects of proton pump inhibitors and electrolyte disturbances on arrhythmias. Int J Gen Med 28: 515-518. doi: 10.2147/ IJGM.S46932

Epstein M, McGrath S, Law F (2006) Proton-pump inhibitors and hypomagnesemic hypoparathyroidism. N Engl J Med 355: 1834-1836

Hoorn EJ, van der Hoek J, de Man RA, Kuipers EJ, Bolwerk C, Zietse R (2010) A case series of proton pump inhibitor-induced hypomagnesemia. Am J Kidney Dis 56: 112-116. doi: 10.1053/j. ajkd.2009.11.019

Kieboom BC, Kiefte-de Jong JC, Eijgelsheim M, Franco OH, Kuipers EJ, Hofman A, Zietse R, Stricker BH, Hoorn EJ (2015) Proton pump inhibitors and hypomagnesemia in the general population: a population-based cohort study. Am I Kidney Dis 66: 775-782. doi: 10.1053/j.ajkd.2015.05.012

Krag M, Perner A, Moller MH (2016) Stress ulcer prophylaxis in the intensive care unit. Curr Opin Crit Care 22: 186-190

Park CH, Kim EH, Roh YH, Kim HY, Lee SK (2014) The association between the use of proton pump inhibitors and the risk of hypomagnesemia: a systematic review and meta-analysis. PLoS One 9: e112558. doi: 10.1371/journal.pone.0112558

Toh JW, Ong E, Wilson R (2015) Hypomagnesaemia associated with long-term use of proton pump inhibitors. Gastroenterol Rep (Oxf) 3: 243-253

William JH, Danziger J (2016) Magnesium deficiency and proton-pump inhibitor use: a clinical review. J Clin Pharmacol 56: 660-668. doi: $10.1002 /$ jcph. 672

William JH, Danziger J (2016) Proton-pump inhibitor-induced hypomagnesemia: current research and proposed mechanisms. World J Nephrol 5: 152-157. doi: 10.5527/wjn.v5.i2.152

Zipursky J, Macdonald EM, Hollands S, Gomes T, Mamdani MM, Paterson JM, Lathia N, Juurlink DN (2014) Proton pump inhibitors and hospitalization with hypomagnesemia: a population-based case-control study. PLoS Med 11: e1001736. doi: 10.1371/journal. pmed.1001736. 